\title{
BIRATIONAL TRANSFORMATIONS OF THE CUBIC VARIETY IN FOUR-DIMENSIONAL SPACE.
}

\author{
By Virgil Snyder (Ithaca, N. Y.).
}

Adunanza del it dicembre 1913 .

Many problems in geometry depend for their solution upon the answer to the question whether the general cubic variety $V$, in linear space of four dimensions $S_{4}$ can be birationally mapped on ordinary space $S_{;}$. While the latter problem has not been solved, various methods have been employed which contribute negative results. I have attempted to determine whether continuous groups of birational transformations exist which leave $V_{3}$ invariant. All the transformations of this kind that I have succeeded in finding, while forming an infinite continuous series, do not form a continuous group. If it can be proved that the transformations here considered are the only ones which leave $V_{3}$ invariant, then it would follow that $V_{3}$ is not invariant under any continuous group of birational transformations, and consequently that it is irrational.

I. The non-singular plane cubic curve cannot be birationally mapped on a straight line, although it is invariant under a continuous group of birational transformations. These transformations are of two kinds, the central and the non-central. The former consist of the projection of the curve upon itself from points upon it, and the latter of the product of two such projections from distinct centers. The former constitute a one-parameter series, one transformation being associated with each point of the curve; the latter constitute a one-parameter group. Given any two central projections $a, b$ and any third $a^{\prime}$. A fourth central projection $b^{\prime}$ can be found such that

$$
a b=a^{\prime} b^{\prime} .
$$

The central is involutorial and has four double elements; the non-central is non-periodic except for particular positions of the centers of the component central projections and has no double elements ${ }^{x}$ ).

1) EM. WEYR, Ueber eindeutige Bexiebungen auf einer allgemeinen ebenen Curve dritter Ordnung [Sitzungsberichte der mathematisch naturwissenschaftlichen Klasse der Kaiserlichen Akademie der Wissenschaften (Wien), Abt. II, Vol. LXXXYII (1883), pp. 837-872]. The results of W'EYR have been corrected and completed by C. SEGRE, Le corrispondenze uniz'oche sulle curv'e ellittiche [Atti della R. Accademia delle Scienze di Torino, Vol. XXIV (1889), pp. 734-756]. 
The transformations of both kinds are CREMOnian, a central projection being expressed as a quadratic inversion of the second hind, that is, a conic $C$ and a point $O$ on it being given, the image $P^{\prime}$ of $P$ is defined as the harmonic conjugate of $P$ as to $O$ and the residual intersection of $O P$ and $C$. If the center $O$ is on the cubic curve the invariant conic is the first polar of the point as to the given cubic curve. When the center is a point of inflexion, the central projection is linear.

2. The corresponding problem for a cubic surface of ordinary space has not been considered, since the surface can be mapped birationally on a plane.

The central projection of the cubic surface upon itself from a point on the surface becomes the Geiser involution when the surface is mapped on a plane by the Grassmann method. The image of a straight line is a rational curve of order eight, with seven triple points. The entire problem is thus transferred to a plane, by cousidering such transformations as arise by combinations of Geiser transformations having different fundamental points.

\section{SI.}

\section{Transformations $\alpha$.}

3. Let $A$ be any point of $V_{3}$ in $S_{+}$. The operation of projecting $V_{;}$upon itself from $A$ will be denoted by the letter $\alpha$, with the condition

$$
x^{2}=\mathbf{I} \text {. }
$$

Its equations may be written as follows. If $A=\left(a_{1}, a_{i}, a_{3}, a_{+}, a_{5}\right)$ and

$$
H(x)=\sum a_{i} \frac{\partial V_{;}}{\partial x_{i}}, \quad V,(a)=0,
$$

and $(v)$ any point in $S_{f}$, then $\left(y^{\prime}\right)$ has the coordinates

wherein

$$
y^{\prime}=y_{1} H(a, y)-a_{1} H(y) \text {, }
$$

$$
H(a, y)=\frac{\mathrm{I}}{2} \sum a_{1} \frac{\partial H}{\partial y_{1}^{\prime}} \text {. }
$$

The transformation $x$ is a quadratic Cremon $t$ transformation, the invariant quadric variety being the first polar of $(a)$ as to $V_{3}$.

Every point in which $H$ meets $V$; is invariant under $x$; it is a surface of order six in $S_{4}$. The operation $x$ is linear when and only when $H(x)$ and $H(a, x)$ have a common linear factor; since $H(a, x)$ is itself linear, it follows that $H(a, x)$ must be a factor of $H(x)$. Thus, the discriminant of $H(x)$ is of rank nor greater than three. This condition requires more independent relations anong the $a_{1}$ than the number of coordinates, hence is possible only for a particular variety $V_{\text {; }}$. Such cases will not be included in the following discussion. 
The number of parameters in $\alpha$ is three, since any point on $V$, may be chosen as vertex. No two transformations $\alpha$ are equivalent.

4. Since $\alpha$ is a quadratic transformation, the section of $V$; made by an $S$; not passing through $A$ is transformed into the complete intersection of $V$, and the quadratic form $V_{2}$ in $S_{4}$, image of $S_{3}$ in $x$. The tangent space to $V_{\text {; }}$ at $A$ meets $S_{\text {; }}$ in a plane which cuts $V_{\text {; }}$ in a cubic curve $C_{;}$. The image of every point of $C_{j}$ is at $A$, hence we may say: The image in $x$ of a section of $V_{;}$by a gerleral $S_{;}$is a surface $\left(V_{2}, V_{3}\right)$ of order six in $S_{+}$, having a triple point at $A$, the tangeris all lying an a cubic conte of an $S_{;}{ }^{2}$ ).

Moreover, the image of any plane section $C_{\text {; }}$ of $V_{\text {; }}$ is a sextic eurve $C_{0}$ lying on a quadric surface $F_{2}$ of $S_{\text {; }}$, as may be seen as follows.

The plane of $C$; and the point $A$ determine an $S_{3}$ which iuts $V_{\text {; }}$ in a cubic surtace $F$; passing through $C$; and through $A$. The cone $K$; having $A$ as vertex and

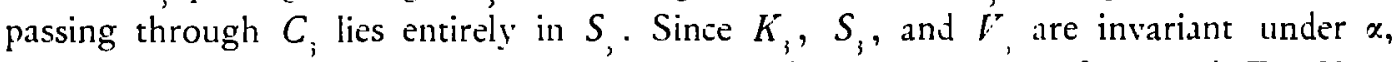
it follows that the image of $C_{;}$in $\alpha$ is the residual intersection of $K$, and $F_{3}$. Since part of their intersection is a complete intersection with a plane, the residual $C_{6}$ must be a complete intersection with a quadric surtace $F_{2}$. The tangent plane to $F_{\text {; }}$ at $A$ meets the plane of $C_{\text {; }}$ in a straight line; this line meets $C_{\text {; }}$ in three points, the image of each of which in is at $A$, hence $A$ is a triple point of $C_{v}$, the tangent lines all lying in one plane.

5. Now consider the product $x_{2} x_{1}$ of two operations $x_{1}$ and $y_{k}$. Here various cases must be considered. On $V_{\text {; }}$ lie $s^{2}$ straight lines. Through every point of $I_{;}$, lie six; they are the six liines of the cubic surface passing through the point of contact and cut from $V_{3}$ by a tangent space. In an arbitrary space $S_{\text {; }}$ lie 27 lines of $V$; lying on the cubic surface iut from $V$ by the $S_{\text {; }}$. Any plane through a line of $V_{\text {; }}$ is a double tangent plane, the points of contact being the points of intersection of the line and the residual conic.

Let $x_{1}=x_{2}=x_{j}=0$ be the equations of a general straight line $p$ on $V_{3}$. The equation of $V^{r}$ may be written in the form

$$
V_{j} \equiv x_{1} \psi_{1}+x_{2} \psi_{2}+x_{j} \psi_{3}=0 \text {, }
$$

in which each $q$, is quadratic in $x_{1}, \ldots, x_{i}$. The tangent $S_{\text {; }}$ to $V_{3}$ at $\left(0,0,0, y_{4}, y_{1}\right)$ on $p$ has the equation

$$
x_{1} \varphi_{1}(y)+x_{2} \varphi_{2}(y)+x_{j} \varphi_{3}(y)=0,
$$

in which $p_{1}(y)=\varphi_{1}\left(\mathrm{o}, \mathrm{o}, \mathrm{o}, y_{1}, y_{3}\right)$. The $S_{3}$ is tangent to $V_{3}$ at one point only.

2) This result could also be ohtained from the theorem of G. FANo \{ Sulle superficie algebriche contenute in una r'arietì iubicia dello spaqio a qualtro dimensioni [Atti della R. Accadenia delle Scienze di Torino, Vol. XXXIX (1904), pp. ;97-6rij\} concerning possible surfaces on $V_{3}$. The result of Fano has been generaliced to apply to $r_{r-1}$ in $S$, without multiple points, namely, that the only possible varieties $V_{r-2}^{r}$ lying on them are complete intersections. See F. SEveri, Una proprietia delle forme algebricbe prive di funti meltipli [Rendiconti della R. Accadenid dei Lincei (Roma), Vol. XV, $2^{\circ}$ semestre [yo6, pp. 6y[-696]. 
Let

$$
\frac{x_{1}}{A_{1}}=\frac{x_{2}}{A_{2}}=\frac{x_{3}}{A_{3}}
$$

be the equations of a plane through $p$. When $y_{4}, y^{\prime}$, are given, $A_{1}: A_{2}: A_{3}$ can be uniquely determined, so that the plane is tangent to $V_{3}$ at $(y)$. If $A_{1}: A_{2}: A_{3}$ are given. two points of tangency are determined, hence every plane through the line is a bitangent plane. There is a $(I, I)$ correspondence between the planes through the line and the pairs of points on the line. The tangent spaces to $V_{3}$ at the points of $p$ envelope a quadratic variety having $p$ for vertex. In every pencil of spaces through $p$ there are two that are tangent to $V_{3}$.

Amoug the straight lines on $V_{3}$ are $\infty^{\prime}$ special straight lines $l$, defined by the property that all the tangent spaces to $V_{\text {; }}$ at points of $l$ have a plane through the line in common. This basis plane touches $I^{r}$, along the entire length of $l$, and intersects $V_{3}$ in a residual line called the conilugate of $l^{3}$ ).

The tangent spaces at points of a special line belong to a pencil; the planes of the pencil and the points of the line $l$ are in $(1,2)$ correspondence. The points of contact form an involution projective with the pencil of spaces. All the planes through $l$ which belong to the same space cut $l_{3}$ in conics which meet $l$ in the same points, hence the conics meet $l$ in points of an involution.

If $x_{1}=x_{2}=x_{3}=0$ is a special line and $x_{2}=0, x_{3}=0$ the basis plane, $p_{1}$ has the form $u_{1} x_{1}+u_{2} x_{2}+u_{3} x_{3}$, in which each $u_{3}$ is linear in $x_{1}, \ldots, x_{5}$. The equation of the tangent space at $\left(0, o, o, y_{+}, y_{;}\right)$has the form

$$
x_{2} \varphi_{2}(y)+x_{;} \psi_{j}(y)=0 \text {. }
$$

The equations of the planes through the line and lying in the $S_{3}$ defined by $x_{j}=m x_{2}$ are of the form $x_{3}+k x_{1}=0, x_{;}=m x_{2}$.

The residual conic in each of these planes meets $l$ in the points of contact of the bitangent space $x_{3}=m x_{2}^{4}$ ).

The centers $A_{1}, A_{2}$ may have the following positions:

(I): not on a common line.

(2): on a line $p$.

(3): on a line $l$.

In case (1), the $\infty^{2}$ plane sections of $V_{3}$ through $A_{1} A_{2}$ remain invariant. The curve of order I2, locus of the point of contact of the $\infty^{\prime}$ tangent spaces through $A_{1} A_{2}$ remains point by point invariant. The operation $\alpha_{1} \alpha_{2}$ can not be replaced by another of the form $\alpha_{1}^{\prime} \alpha_{2}^{\prime}$.

The image of $\left(V_{3}, S_{3}\right)$ by $\alpha_{1}$ is a sextic surface $\left(V_{3}, V_{2}\right)$ of $S_{4}$, having a triple

3) F. ENRIQUes, Sugli spazi pluritangenti delle z'arieti cubiche generali appartenenti allo spazio a 4 dimensioni [Giornale di Matematiche di Battaglini, Vol. XXXI (1893), pp. 3I-35].

4) G. FANO, Ricerche sulla varieta iubica generule dello spaiio a quattro dimensioni e sopra $i$ suvi spuzi pluritangenti [Annali di Matematica pura ed applicata, Series III, Vol. X (1904), pp. 25I-285]. 
point at $A_{1}$ and no other multiple points. The image of $\left(V_{3}, V_{2}\right)$ in $x_{2}$ is a complete intersection $\left(V_{3}, V_{4}\right)$ of order 1 2, having a sixfold point $A_{2}$, a triple point at the image of $A_{1}$ in $\boldsymbol{\alpha}_{2}$, and no other point singularities.

The image of the $C_{6}$ on an $F_{2}$ having a $P_{3}$ at $A_{1}$ in $\alpha_{2}$ may be found as follows. The lines joining $A_{2}$ to points of $C_{6}$ from a twodimensional cone $K_{6}$ of $S_{4}$, of order six. Any $S_{3}$ through $A_{2}$ cuts six generators from $K_{0}$, on each of which lies one point of the image of $C_{6}$ in $x_{2}$, besides those at $A_{2}$. The tangent $S_{3}$ to $V_{3}$ at $A_{2}$ meets $C_{6}$ in six points, the image of each of which is at $A_{2}$. Hence the image of $C_{6}$ is a curve of order trielve, $C_{12}$ having a six fold point at $A_{2}$, a triple point at the image of $A_{\text {, }}$ in $x_{2}$, and no other point singularities. Moreover, $C_{12}$ is a complete intersection of $V_{3}$, of $r_{2}$, the image of $S_{3}$ in $\alpha_{2}$, and $K_{2}$, the three dimensional quadric cone obtained by projecting $F_{2}$ from $A_{2}$.

Hence the operation $\alpha_{1} \alpha_{2}$ can not be replaced by another of the form $\alpha_{1}^{\prime} \alpha_{2}^{\prime}$, unless possibly when $A_{1}^{\prime}$ lies on the line $A_{1} A_{2}$.

Let the line $A_{1} A_{2}$ intersect $V_{3}$ in a third point $A_{;}$. The only possible combinations are

$$
x_{1} x_{2}=\alpha_{3} x_{1} \text { and } x_{1} x_{2}=x_{2} x_{3} \text {. }
$$

The operation $\alpha_{1} \alpha_{2}$ transforms the point $A_{2}$ into $A_{1}$, while the operation $\alpha_{3} x_{1}$ transforms $A_{2}$ into the first tangential of $A_{1}$. If this point coincides with $A_{1}$ for every section through $A_{1} A_{2}$, every tangent to $V_{3}$, at $A_{\text {; }}$ must have three point contact. In this case the polar quadric of $A_{1}$ is composite and contains the tangent space to $V_{3}$ at $A_{\mathrm{I}}$ as one component. This was seen to be possible only for particular varieties $V_{3}$, hence in the case under consideration no such points exist. Similarly for the hypothesis $\alpha_{1} \alpha_{2}=\alpha_{2} \alpha_{3}$. This proves the proposition.

(2). Any plane through $p$ cuts from $V_{3}$ a conic cutting $p$ in two points $P, Q$. In this plane $A_{1} A_{2}$ may be replaced by any pair of points $B_{1} B_{2}$ on $p$ which with $P, Q$ have the same cross-ratio. But as the plane turns about $p$, the points $P, Q$ can come into coincidence with any pair of points whatever of $p$, it follow's that it is impossible that the cross-ratio $\left(A_{1} A_{2} P Q\right)$ shall always be equal to $\left(B_{1} B_{2} P Q\right)$, hence the operation $\alpha_{1} \alpha_{2}$ is not equivalent to $B_{1} B_{2}$ for other planes.

(3). The same argument applies to a special line except for the case in which $A_{1}, A_{2}$ are points of contact of a bitangent plane. In this caşe $\left(\alpha_{1} x_{2}\right)^{2}=\mathrm{I}$ and the centers $A_{1} A_{2}$ may be replaced by any other pair of points in the involution formed by the bitangent spaces ${ }^{5}$ ). Hence.

The only involutions $\left(\alpha_{1} \alpha_{2}\right)^{2}=1$ on $V$, are those on the special lines, one on each line $l$. One center $A_{1}$ may be assumed at will, and the other is uniquely defined.

We have established the two results.

The transformation $\alpha_{1} \alpha_{2}$ contains six parameters. The transformations $\alpha_{1} \alpha_{2}$ cannol form a continuous group, nor can they include any subset which forms a continuous group.

5) C. Stéphanos, Mémoire sur la représentation des bomographies binaires par des points de l'espace ar'ec application a l'stude des rotations spheriques [Mathematisihe Annalen, Vol. XXII (1883), pp. 299-367]. 
6. Now consider the product of three projections $\alpha_{1}, \alpha_{2}, \alpha_{3}$. The plane determined by the three vertices remains invariant, and in this plane the product can be replaced by one central projection, having four invariant points. It has no invariant points except those in the plane $A_{1} A_{2} A_{j}$. If another operation $\alpha_{1}^{\prime} \alpha_{2}^{\prime} \alpha_{3}^{\prime}$ can be found equivalent to $\alpha_{1} \alpha_{2} \alpha_{j}$, the vertices $A_{1}^{\prime} A_{2}^{\prime} A_{3}^{\prime}$ must lie in the plane $A_{1} A_{2} A_{3}$.

Consider the image of the section $\left(V_{3}, S_{3}\right)$ made on $V_{3}$ by any $S_{3}$, when transformed by $\alpha_{1} \alpha_{2} \alpha_{3}$. It has been seen that the image of $\left(V_{3}, S_{3}\right)$ in $\alpha_{1} \alpha_{2}$ is a surface of $S_{4}$ of order 12, the complete intersection of $V_{3}$ with $V_{4}$. This surface is transformed by $\alpha_{3}$ into $\left(V_{3}, V_{3}\right)$ of order 24 ; it has a conical point of order I 2 at $A_{3}$, a point of order 6 at the image of $A_{2}$ in $\alpha_{5}$, a triple point at the image of $A_{1}$ in $\alpha_{2} \alpha_{3}$, and no other point singularities. If the same section were transformed by $\alpha_{1}^{\prime} \alpha_{2}^{\prime} \alpha_{3}^{\prime}$, the resulting surface would have similar singularities at $A_{3}^{\prime}$, etc., hence when $A_{1} A_{2} A_{3}$ do not lie by twos on lines of $V_{3}$, no operation $x_{1}^{\prime} \alpha_{2}^{\prime} x_{3}^{\prime}$, can be found which is equivalent to the given one $\alpha_{1} \alpha_{2} \alpha_{3}$.

It has been seen that the image of a general plane section in $x_{1} x_{2}$ is a curve $C_{12}$ of $S_{+}$which is also a complete intersection. The image of $C_{12}$ in $x_{3}$ is not a complete intersection, nor can any general conclusions be made concerning images of curves in $S_{4}$.

Among the lines which lie on $V_{3}$ and meet a given special line of $V_{3}$ are 28 special lines. These are arranged in 14 pairs, such that the lines of a pair intersect each other. The triangle formed by three special lines has points of contact of a tritangent space for vertices ${ }^{6}$ ).

Let $A_{1}, A_{2}, A_{3}$ be the vertices of a triangle of special lines. The operation $\alpha_{1} \alpha_{2} \alpha_{3}$ is equivalent to that expressed by any permutation of these same factors, or to such combinations as $\alpha_{1}^{\prime} \alpha_{2}^{\prime} \alpha_{3}^{\prime}$, in which $A_{2}^{\prime}, A_{3}^{\prime}$ are the points of contact of any bitangent space through $A_{2} A_{;}$. It is also involutional.

When $A_{1}, A_{2}, A_{3}$ are vertices of a triangle the only involutions are those defined by the points of contact of tritangent spaces.

Finally, if $A_{1}, A_{2}, A_{3}$ all lie on a line and $\alpha_{1}^{\prime} \alpha_{2}^{\prime} \alpha_{3}^{\prime}$ is equivalent to $\alpha_{1} \alpha_{2} \alpha_{3}$, evidently $A_{1}^{\prime}, A_{2}^{\prime}, A_{3}^{\prime}$ must lie on the same line. Three cases arise:

The line $A_{1} A_{2} A_{3}$ is $(a)$ not on $V_{3} ;(b)$ a non-special line $p ;(c)$ a special line 1 . In case $(a)$ the only possibilities are

$$
\alpha_{2}^{\prime} \alpha_{2}^{\prime} \alpha_{3}^{\prime}=\alpha_{2} \alpha_{3} \alpha_{1} \quad \text { or } \quad \alpha_{1}^{\prime} \alpha_{2}^{\prime} \alpha_{3}^{\prime}=\alpha_{3} \alpha_{1} \alpha_{2} \quad \text { or } \quad \alpha_{1}^{\prime} \alpha_{2}^{\prime} \alpha_{3}^{\prime}=\alpha_{3} \alpha_{2} \alpha_{1} .
$$

In any case this is possible only when the tangents at two of the vertices meet on the curve of section for every plane through the line, but this is not possible unless the tangent spaces intersect on $V_{3}$, and the general $V_{3}$ does not contain a plane. In cases $(b),(c)$ no equivalence is possible from Nos. 4 and 5 . Hence we may say.

The operation $\alpha_{1} \alpha_{2} \alpha_{3}$ defines a nine-parameter transformation. The transformations do not form a continuous group nor include any continuous group with fewer parameters.

6) G. Fano, loc. (it. 4). 
7. Now consider the operation $x_{1} \alpha_{2} \alpha_{3} x_{4}$. Various cases are to be considered, according as the space of fewest dimensions which contains $A_{1}-A_{4}$ is an $S_{3}$, and $S_{2}$ or an $S_{1}$. If $A_{1}-A_{4}$ do not lie in a plane, and four points $A_{1}^{\prime} A_{2}^{\prime} A_{3}^{\prime} A_{4}^{\prime}$ can be found such that $x_{1}^{\prime} \alpha_{2}^{\prime} x_{3}^{\prime} x_{+}^{\prime}=\alpha_{1} \alpha_{2} \alpha_{j} x_{+}$, then the smallest space through $A_{1}-A_{+}$is also an $S_{3}^{\prime}$. If $S_{3}^{\prime} \neq S_{3}$, the $F_{3}$ cut from $V_{3}$ by $S_{3}$ is invariant and the $F_{3}^{\prime}$ cut from $S_{3}^{\prime}$ is invariant. But $F_{3}^{\prime}$ is transformed into an $F_{0}^{\prime}$ by $x_{1}$, ete., which cannot return to $F_{3}^{\prime}$ unless $\alpha_{1} x_{2} \alpha_{3} x_{4}$ is the identity, by No. 5. But in case $A_{1}-A_{+}$are not coplanar $\alpha_{1} \ldots \alpha_{+}$cannot be the identity, by No. 4 . Hence this cise is impossible. If $S_{3}^{\prime}=S_{3}$ we can proceed exactly as in No. 7 to obtain the following results.

The operation $\alpha_{1} \alpha_{2} x_{3} \alpha_{4}$ is a 12 parameter transformation. These transformations do not form a continuous group nor include a continuous group of fewer parameters.

The only involution of the form $x_{1} x_{2} x_{3} x_{+}$is defined $b y$ the points of contact of a quadritangent space. $V_{\text {; }}$ has 495 such quadritangent spaces.

8. It is now easy to generalize.

That the operation $\prod_{j=1}^{k} x$, is a transformation involving $3 k$ parameters, that the transformations do not form a continuous group nor include a continuous group with feuer parameters. The only transformations that are of finite period are the incolutions $\alpha_{1}, x_{1} \alpha_{2}$, $\alpha_{1} \alpha_{2} \alpha_{3}$ and $\alpha_{1} \alpha_{2} \alpha_{3} \alpha_{4}$ of multitangent spaces.

\section{$\S 2$.}

\section{The Transformations $\pi$.}

9. A second birational transformation leaving $V_{3}$ invariant may be defined as follows. Pass a plane $S_{2}$ through a line $p$ of $V_{3}$. The residual intersection of $V_{3}$ by $S_{2}$ is a conic. Let $P$ be the pole of $p$ as to the conic. The transformation is the harmonic homology in each plane $S_{2}$ determined by $p$ and $P$.

This transformation $\pi$ is also Cremonian. When $p$ is an ordinary line of $V_{3}$, the $\infty^{2}$ planes through it determine $\infty^{2}$ pairs of points upon it and also $\infty^{2}$ positions of $P$. Hence the locus of $P$ is a rational surface in $S_{+}$, since any plane through $p$ meets it in just one point not on $p$.

To determine the equations of the transformation, let $x_{1}=0, x_{2}=0, x_{3}=0$ be the equations of $p$.

$$
V_{3}=x_{1} p_{1}+x_{2} \varphi_{2}+x_{3} \varphi_{3}
$$

The equations of a plane through $p$ are

$$
\frac{x_{1}}{\lambda}=\frac{x_{2}}{\mu}=\frac{x_{3}}{v}
$$

and the residual conic is defined by

$$
\lambda \varphi_{1}\left(x_{1}, \frac{\mu x_{1}}{\lambda}, \frac{v x_{1}}{\lambda}, x_{4}, x_{s}\right)+\mu p_{2}+v \varphi_{3}=0 .
$$


By putting $x_{1}=0$ we have a quadratic equation $x_{4}: x_{\text {; }}$ whose roots define the points of intersection of $p$ and the conic. Let these points be

$$
K^{\prime} \equiv\left(\mathrm{o}, \mathrm{o}, \mathrm{o}, k_{4}^{\prime}, k_{\xi}^{\prime}\right), K^{\prime \prime} \equiv\left(\mathrm{o}, \mathrm{o}, \mathrm{o}, k_{4}^{\prime \prime}, k_{\varsigma}^{\prime \prime}\right) \text {. }
$$

The equations of the tangents to the conic at $K^{\prime}, K^{\prime \prime}$ are of the form

$$
\begin{aligned}
& \left(A k_{4}^{\prime}+B k_{5}^{\prime}\right) x_{1}+\left(B k_{+}^{\prime}+C k_{5}^{\prime}\right) x_{+}+\left(D k_{4}^{\prime}+E k_{5}^{\prime}\right) x_{5}=0 \\
& \left(A k_{+}^{\prime \prime}+B k_{5}^{\prime \prime}\right) x_{1}+\left(B k_{+}^{\prime \prime}+C k_{5}^{\prime \prime}\right) x_{4}+\left(D k_{+}^{\prime \prime}+E k_{5}^{\prime \prime}\right) x_{;}=0 .
\end{aligned}
$$

The coordinates of the point of intersection are all multiples of $\left(k_{4}^{\prime} k_{3}^{\prime \prime}-k_{;}^{\prime} k_{4}^{\prime \prime}\right)$. Discarding this factor we have

$$
x_{1}=(B E-C D), \quad x_{4}=(D B-A E), \quad x_{5}=\left(A C-B^{2}\right),
$$

in which $A, B, C$ are distinct linear functions of $i, \mu, \vee$ and $D, E$ are independent quadratic functions of $\lambda, \mu, v$. The coordinates of $P$ are thus

$$
P \equiv\left(\lambda g_{2}, \mu g_{2}, \vee g_{2}, \psi_{3}, f_{3}\right)
$$

wherein $\psi_{1}, f_{i}, g_{i}$ are polynomials of degree $i$ in $\lambda, \mu, v$. The equations of the locus of $P$ are therefore

$$
\begin{aligned}
& \psi_{3}\left(x_{1}, x_{2}, x_{3}\right)=x_{4} g_{2}\left(x_{1}, x_{2}, x_{3}\right), \\
& f_{3}\left(x_{1}, x_{2}, x_{j}\right)=x_{1} g_{2}\left(x_{1}, x_{2}, x_{3}\right) .
\end{aligned}
$$

The section of this surface by an arbitrary $S_{;}$is a space curve of order nine. An $S_{\text {; }}$ through $p$ cuts it in $p$ counted six times and in a space cubic curve cutting $p$ twice.

The equations of the transformation are found by determining $\left(x^{\prime}\right)$, the harmonic conjugate of a given point $(x)$ as to $P$ and the intersection of $(x) P$ with $p$. They are

$$
\begin{gathered}
\sigma x_{1}^{\prime}=-g_{2} x_{1}, \sigma x_{2}^{\prime}=-g_{2} x_{2}, \sigma x_{j}^{\prime}=-g_{2} x_{j}, \\
\sigma x_{4}^{\prime}=2 \psi_{j}\left(x_{1}, x_{2}, x_{j}\right)-g_{2} x_{+}, \sigma x_{j}^{\prime}=2 f_{j}\left(x_{1}, x_{2}, x_{j}\right)-g_{2} x_{5} .
\end{gathered}
$$

The line $p$ is fundamental. An arbitrary $S_{3}$ is transformed into a cubic cone of the first hind having the point in which $S_{\text {; }}$ meets $p$ as vertex. The line $p$ is a double line

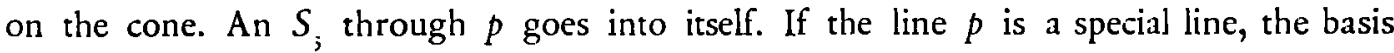
plane is fundamental. We may now put

$$
\begin{gathered}
V_{3}=x_{1} p_{a}+x_{2} \varphi_{k}+x_{3} p_{c}=0, \\
p_{a}=\sum a_{1 k} x_{1} x_{k}, \quad p_{k}=\sum b_{1 k} x_{1} x_{k}, \quad \varphi_{1}=\sum c_{1 k} x_{1} x_{k} .
\end{gathered}
$$

If the basis plane is $x_{2}=0, x_{j}=0$, then $a_{4+}=a_{45}=a_{3 j}=0$. In this case we have

$$
\begin{aligned}
& A=\mu b_{44}+\nu c_{4+}, \quad B=\mu b_{45}+\nu c_{4 j}, \quad C=\mu b_{55}+\nu c_{5 j} . \\
& D=\lambda\left(\lambda a_{41}+\mu b_{41}+\nu c_{+1}\right)+\mu\left(\mu b_{42}+\nu c_{42}\right)+v^{2} c_{4 j}, \\
& E=\lambda\left(\lambda a_{s 1}+\mu b_{51}+\nu c_{51}\right)+\mu\left(\mu b_{52}+\nu c_{52}\right)+\nu^{2} c_{53} .
\end{aligned}
$$

In case of a non special line $\pi$ cannot be expressed in terms of the transformations $\alpha$.

Consider the image of an arbitrary $S_{\text {; }}$ by $\pi_{1} \pi_{2}$. By $\pi_{1}, S_{j}$ is transformed into a three dimensional cubic cone, having a point on $p_{1}$ as vertex and the line $p_{1}$ as a double line. By $\pi_{z}$ this cone is transformed into a variety of $S_{t}$, containing a double 
curve and containing $p_{2}$ as a sixfold line. If $p_{1}, p_{2}$ intersect, the double curve is replaced by a simple plane curve lying in a tangent $S_{2}$ to $V_{3}$, but $p_{2}$ is still a six fold line. Hence no pair of operators $\pi_{1} \pi_{2}$ can be replaced by another pair. In the same way it is seen that the product of three operations $\pi_{1} \pi_{2} \pi_{3}$ ean not be replaced by any other product of three, etc., hence the operations $\pi$, can not form a continuous group nor any combination of them generate a continuous group.

Moreover, by combining the preceding results, we see that no combination of operations $x, \pi$ can form a continuous group.

In case of a special line 1

$$
\pi=x_{1} x_{2}=x_{2} x_{1},
$$

$A_{1}, A_{2}$ being the points of contact of any bitangent space on $l$.

\section{$\int 3$.}

\section{The variety $\sum x_{1}^{3}=0$.}

10. It would not be appropriate to this discussion to consider all the special or particular forms of the cubic variety, but one form of considerable interest was mentioned by FANO ${ }^{7}$ ).

The variety $V \equiv \sum x_{1}^{i}=0$ contains no planes and no double points, but has the maximum number (thirty) of points at which the tangent space intersects the variety in a cubic cone. If $i, k, l, m, n$ represent any permutation of the intergers $\mathrm{I}$, $2,3,4,5$, and $\omega$ is any number which satisfies the equation $\omega^{3}=\mathrm{I}$, the equations of these particular spaces are of the form $x_{1}+\omega x_{k}=0$ and section cut from the variety is $x_{1}^{3}+x_{m}^{3}+x_{n}^{j}=0$. It was shown by Fano that the variety is invariant under a collineation group of order $3^{+} 5$ ! made up of replacing each $x_{1}$, by $\omega x_{1}$, each coefficient being independent, and of the permutation of the coordinates.

These transformations can all be expressed in terms of projections from the vertices of the cones. Thus, from the point $(0,0,0, \omega, I)$ the operation $x$ has the equations

$$
x_{1}^{\prime}=x_{1}, \quad x_{2}^{\prime}=x_{2}, \quad x_{3}^{\prime}=x_{j}, \quad x_{4}^{\prime}=\omega x_{j}, \quad x_{5}^{\prime}=\omega^{2} x_{4} .
$$

I wish to thank Professor Francesco Severi, of the University of Padua, for suggesting this problem and for much kindly assistance in connection with it.

Cornell University, September 1913 .

VIRGIL SNYDER.

7) G. Fano, Sopra una v'arield cubicu particolare dello spazio a quattro dimensioni [Rendiconti del R. Istituto Lombardo di Scienze e Lettere (Milano), Series II, Vol. XXXVII (I904), pp. 554-566]. 